\title{
Philosophiques
}

\section{Essai de métaphysique (Notes et jalons)}

\section{Jean Goulet}

Volume 6, numéro 2, octobre 1979

URI : https://id.erudit.org/iderudit/203128ar

DOI : https://doi.org/10.7202/203128ar

Aller au sommaire du numéro

Éditeur(s)

Société de philosophie du Québec

ISSN

0316-2923 (imprimé)

1492-1391 (numérique)

Découvrir la revue

Citer cette note

Goulet, J. (1979). Essai de métaphysique (Notes et jalons). Philosophiques, 6(2), 363-386. https://doi.org/10.7202/203128ar d'utilisation que vous pouvez consulter en ligne.

https://apropos.erudit.org/fr/usagers/politique-dutilisation/ 


\title{
ESSAI DE MÉTAPHYSIQUE
}

\author{
(Notes et jalons) \\ par Jean Goulet
}

\section{Avant-propos}

Les présentes Notes ont un caractère à la fois incomplet et systématique. Elles accompagnent un discours dont le propre est de vouloir dire une vérité difficile associée à l'émergence d'un sens, donc à notre liberté de le refuser ou d'y consentir. On verra mieux, d'ailleurs, en cours de route, que ce sens n'est pas distinct de la liberté : mystère de l'existence de "quelque chose plutôt que rien ", mystère de l'existence de l'homme, qui n'a pas choisi d'être, mais qui est libre de chercher ou non à comprendre.

C'est pour cela que "la métaphysique n'est pas faite", que chaque homme est invité à la reprendre toujours à son propre compte, à tous les moments de l'histoire. Elle est la vie même d'un acte libre de connaissance. Acte personnel et acte à plusieurs, où l'assurance de soi et l'unanimité sont toujours difficiles à réaliser. D'où, également, une bibliograpbie indéfiniment ouverte, étriquée, où les témérités de la négation voisinent avec celles de l'affirmation, et où, là encore, chacun choisit ce qu'il lui plaît de lire.

\section{Préliminaires}

1. Définition nominale de la métaphysique : connaissance (pour autant qu'elle est possible) du sens que l'bomme attribue à la totalité.

connaissance : non "science" au sens moderne du mot; mais science quand même, i.e. savoir organisé le plus rigoureux possible, visant une " cohérence maximale ", en dépit de la diversité de l'être, de la faiblesse humaine, et du mal. Ce savoir dit vouloir et pouvoir de quelque manière "tenir ensemble, dans le sens, ce qui, séparé, en serait dépourvu».

sens : ce que l'intelligence reconnaîtrait comme vrai et bon, i.e. conforme à ce qui est, et ayant raison d'être.

l'homme : ce savoir serait forcément "humain", ne tiendrait ni du merveilleux ou du prodige, ni d'une révélation, mais de la mesure humaine.

totalité : il s'agit d'un savoir global, dont les parties éclaireraient l'ensemble et seraient en même temps éclairées par l'ensemble.

1.1. Cette définition rejoint ce qu'Aristote dit de la "sagesse ", au livre $1^{\text {er }}$, chapitre 2, de sa Métaphysique, et où il en énumère les six caractères. 
Dans ce texte, l'aspect à la fois possible et surbumain d'une telle entreprise est bien indiqué. De même, pour Aristote, l'étonnement, qui est au départ de la connaissance, demande de se terminer par l'étonnement contraire, qui est de comprendre la richesse de ce qui paraissait d'abord aporétique.

1.2. Autorité de saint Thomas, confirmant celle d'Aristote. "Ainsi que l'enseigne le Philosophe (Aristote) dans sa Politique, lorsque des choses sont en grand nombre ordonnées à une seule, il faut que l'une d'entre elles serve de régulatrice, de directrice, et que les autres soient mesurées, dirigées. C'est ce qui se manifeste dans l'union de l'âme et du corps, où tout naturellement l'âme commande et le corps obéit. Il en est également de même des puissances de l'âme, où l'irascible et le concupiscible obéissent à la raison en vertu d'un ordre inscrit dans la nature des choses. Or les sciences et les arts sont tous ordonnés à une même fin, à savoir l'accomplissement de l'bomme, c'est-à-dire son bonbeur. Il faut donc que l'une d'entre ces disciplines assume la direction des autres; ce qui fait alors qu'on l'appelle à bon droit la sagesse, car c'est du sage que l'ordre dépend ». (Prologue au commentaire à la Métaphysique d'Aristote).

1.3. Autorité d'Heidegger (nettement plus "phénoménologique ") : "Chaque question métaphysique embrasse toujours l'ensemble de la problématique. Elle est chaque fois l'ensemble lui-même. Mais alors, aucune question métaphysique ne peut être posét sans que le questionnant, comme tel, ne soit lui-même compris dans la question, c'est-à-dire pris dans la question ". (Qu'est-ce que la métaphysique? )

Ceci rejoint de même le mot de G. Marcel: "Qui suis-je, moi qui questionne sur l'être?" (Pasitions et approches, etc.)

2. Le mot " métapbysique ». (Cf. Pierre Aubenque, Le probleme de l'être chez Aristote, au début). Le mot n'est pas d'Aristote, encore moins de Platon ou des pré-socratiques, mais serait d'Andronicos de Rhodes ( 60 av. J.-C.), classant les traités d'Aristote. Chez ce dernier, la métaphysique s'appelle philosophie première, ou théologie, ou simplement philosophie, considérée comme sagesse. Ce qui confirmerait quelle est l'aspect selon lequel elle est à la fois plus proprement intuitive et unitaire.

C'est le mot "métaphysique " qui a été retenu au moyen âge. Il culmine dans la critique de Kant, qui en annonce le crépuscule.

3. Quatre des nombreux sens modernes du mot " métaphysique ».

Francis Bacon : la sommation la plus intuitive possible des connaissances physiques, portant sur les causes formelles et finales des êtres naturels. (Voir The Advancement of Learning, II, 7). Pour Bacon, ce savoir est distinct de la philosophia prima, qui, elle, porte sur les principes et axiomes communs à toutes les sciences.

Descartes : un départ absolu du moi réfléchissant sur sa pensée. Voir Discinurs de la métbode et Méditations sur la philosopbie première.

Kant : l'établissement critique (a priori) des rapports entre les conditions de la possibilité de l'expérience phénoménale (connaissance du monde) et la tendance de l'entendement à dépasser l'expérience (par les idées transcendentales de monde, d'âme et de Dieu), l'une et l'autre (c'est-à-dire l'expérience et 
la tendance) étant données à l'homme par la nature en vue d'une fin morale (l'agir libre) à laquelle il est lié.

La métaphysique ainsi comprise n'a pas à proprement parler d'objet (le noumène étant inconnaissable), mais un objectif, une fonction. Aussi dit-il que «par sa fin, son utilité, elle est une institution de la nature». (Cf. Prolégonienes à toute métaphysique future etc.).

On comprend aisément pourquoi il est dit que Kant a mis fin à la métaphysique. Sa métaphysique, qui est essentiellement une "critique ", a effectivement conduit à une "mort" des prétentions métaphysiques, v.g. chez Hegel, Nietzsche, Marx, dans le positivisme, dans une certaine philosophie dite analytique, dans une certaine philosophie dite structuraliste, dans certaines philosophies de l'imaginaire, dans ce qu'on a appelé récemment "fuite en avant".

Heidegger : le fait de l'homme même, absolument et comme donateur de sens.

4. Quatre objections à la possibilité et/ou à la légitimité de la métaphysique.

a) l'existence irrémédiable du mal ; la métaphysique est alors irrecevable.

b) la complexité incommensurable du réel; elle est alors irréalisable.

c) la science pourvoit à tout le sens; elle est alors superflue.

d) l'homme (le surhomme) pourvoit à tout le sens ; elle est alors superflue.

Réponse provisoire à ces objections.

5. Distinction préliminaire utile entre trois types fondamentaux de connaissance du réel :

a) immédiate, perceptuelle, pittoresque.

b) scientifique, dite "vérifiée ", dépendant d'observations méthodiques, partiellement réflexive.

c) philosophique (métaphysique), principalement intuitive-réflexive.

(Cf. Le caractère positivo-négatif de la métaphysique).

6. Méthode suivie dans la présente démarche:

a) approche globale : situation noétique de l'homme ; passage à l'immanence et sortie du paradoxe ; passage à la transcendance ;

l'aspect déterminant du "projet", de la fin ;

le problème du destin de l'homme; établissement de six thèmes-outils ; reconnaissance de la circularité de la démarche.

b) pondération historique des six thèmes : découvertes, oublis, explicitations, récurrences.

c) analyse des fondements, i.e. des thèmes-outils considérés cette fois comme "genres suprêmes " d'une connaissance métaphysique.

Justification de la méthode adoptée.

7. Éléments de bibliographie. 


\section{Approche globale}

8. Situation noétique fondamentale de l'bomme.

Il n'y a pas de fait métaphysique simple, parfaitement clair, percutant, et qui par là serait primitif. Contrairement au goût que la pensée moderne nous en a donné, il n'y a pas, en philosophie, en métaphysique, de départ absolu, d'iclée-daire-principe-absolu, ni non plus d'expérience cruciale.

Il y a là un paradoxe fondamental : quand paraît l'homme, paraît l'exigence de parfaite clarté, c'est-à-dire d'intelligence parfaite, d'évidence. Mais en mème temps paraît labsence de parfaite clarté, d'évidence.

Platon représentait cette situation par le mythe de la caverne. Les Grecs en général (et Socrate avec eux), avaient parlé d'un curieux impératif de " se bien connaître soi-même". Là pour eux semblait résider le mystere central, à la mesure de l'homme; d'un homme protégé par le "sens de la mesure". Aristote reprend la même idée: "Ce que les yeux des chauves-souris sont en effet à l'éclat du jour, l'intelligence de notre âme l'est aux choses qui sont de toutes les plus naturellement évidentes ". (Mét. II).

8. 1. Ce paradoxe s'exprime (entre autres choses) en ce que ceux qui contestent la possibilité d'un discours métaphysique, l'affirment aussi, à leur manière : v.g. en "dénongant " l'absurdité du mal ; en "reconnaissant " la possibilité de la science, en "récupérant "l'homme. Il s'exprime en second lieu en ce que ceux qui disent possible le discours métaphysique ont tous reconnu son caractère difficile, inachevé, etc. Par exemple: Aristote (qui parle de facilité-difficulté de la métaphysique) ; l'ensemble du moyen âge, qui alors a recommandé prudemment la "croyance ", à défaut de philosophie parfaite et accessible à tous; Descartes, recourant à la "garantie" de Dieu; Jobn Locke, dans des textes remarquablement empreints de sagesse ; Kant : "La raison humaine a cette destinée singulière. . . etc. "; Bergson, qui parle de "renversement " de la connaissance, comme Platon parlait de "conversion"; Jaspers, qui aboutit à une sagesse " déchirée ", "suspendue "; G. Marcel, qui parle de "mystère ontologique ", etc., etc..

8.2. S'il n'y a pas de fait métaphysique simple, au départ, il y a donc bien une situation de fait dont il faut partir. Notre mode de connaitre n'est ni logico-mathématique ni divin mais celui d'une "conscience " qui se construit progressivement, tout comme nous la prenons en charge chaque matin.

8.3. Le paradoxe métaphysique n'est rien d'autre que celui qui est recrmn explictement comme situation quotidienne vécue implicitement par l'homme. L'existence, la pratique humaines, quotidiennement, sont paradoxales. C'est l'habitude et le souci qui nous les font oublier.

V.G. la naissance d'un enfant, le rire, la connaissance, la liberté, la situation "cosmique" de l'homme (la Terre lancée dans l'espace...), la mort quotidienne, etc.

La réflexion métaphysique fixe le paradoxe de notre situation, et en fait son point de départ.

9. Elle dit alors (après l'avoir longtemps observé), que l'bonmme ne vit pas de vérités démontrées, mais 
1) de confiance dans la nature;

2) de lucidité, dans l'instant, i.e. de conscience ;

3) de projets.

Bien apercevoir les trois parties de cette "immanence ": Nature; / moi humain conscient, présent à lui-même et à la Nature; / élan d'un projet.

Voir en même temps qu'on passe ainsi d'une situation à une réalité (qu'on appelle ici "immanence ", où l'on aperçoit pourquoi au départ on était en "situation": l'bomme est au centre, entouré.

9.1. Ces trois thèmes ont tendance à correspondre aux trois aspects de la temporalité : "tendance ", car il faut bien reconnaître que la Nature n'est pas que "passé », elle est aussi du "présent"; et l'homme n'est pas non plus que conscience du présent, il est relié en lui-même à sa genèse, à son passé, à la Nature ; et d'ailleurs la Nature a eu un projet, à ce qu'il semble, qui serait l'homme même, l'apparition de l'esprit ; et l'homme a lui-même un projet. Mais il est bon que l'on voie ainsi, d'abord dissociés dans le temps, ces trois aspects de l'existence bumaine.

9.2. Apercevoir comme distincts ces trois aspects, c'est apercevoir une complexité de composition. Le réel (immanent) est composé. Au départ, au contraire, la complexité en était une de situation, d'enchevêtrement de la question.

9.3. Mais il faut tout autant apercevoir l'unité de ces trois thèmes, et le fait que l'homme y occupe une position médiane particulière, par sa lucidité, par l'instant, qu'il fait apparaître.

Importantes "Pensées ", ici, de Pascal, notamment le \#72, appelé Disproportion de l'homme. "L'homme perçoit quelque apparence du milieu des choses $"$.

Cette idée de position médiane de l'bomme ne va pas du tout de soi, quoiqu'elle parût familière à la conscience du XVIrème siècle. Il faut donc décider si on lui accorde un sens ou pas. Ce qui est sûr, c'est que la présente démarche, elle, a fondamentalement cette vue optimiste de la place à la fois modeste et exceptionnelle de l'homme dans la Nature. Les propos de Pascal paraissent ici décisifs. Ils pourraient être aussi corroborés par une vision "anthropologique " de l'évolution.

Biblio. : MAX SCHELER : La situation de l'bomme dans le monde, BERGSON : L'Évolution créatrice, tout le $3^{\text {ème }}$ chapitre, et surtout les pages 252-271.

9.4. Noter que si l'homme est voulu par la Nature, il est impossible de le prouver, du fait même que rien, dans la Nature, avant l'homme, n'est habilité pour le dire. L'homme, pour le savoir et le dire, ne peut compter que sur lui-même. Et c'est là, sans doute, une de ces choses " les plus naturellement évidentes " (Aristote) qu'il est difficile à notre esprit de voir. Et la science, d'autre part, est tenue, par sa méthode même, de mettre une telle idée entre parenthèses. (CF. G.G. SIMPSON, L'évolution et sa signification).

9.5. Ici apparât davantage que tenir un discours métaphysique, c'est-à-dire sur le sens des choses, aussi bien que de l'homme, du temps, etc., est non seulement difficile, mais une difficulté qui définit l'homme même, et par là 
un défi à relever. Pour les anciens stoïciens (v.g. Épictète), et aujourd'hui pour un philosophe comme Lavelle, la philosophie se ramène ainsi à un pouvoir d'AFFIRMATION qui ne relève que de lui-même. (Biblio. : A. FOREST, Du consentement à l'être).

10. Rappel : La temporalité :

- antértorité de la Nature (2ème thème-outil)

- présence de la conscience (3ène thème-outil)

- projet et mouvement de l'bomme (4'ème thème)

Au plan de cette immanence, à travers l'homme conscient, l'être se définit ce qui fut. est, sera ; (et non l'être vide).

10.1. Noter que pour Platon, la métaphysique comme savoir spécifique est encore inexistante. Que pour Aristote, elle existe, mais son statut est encore indéterminé ; son objet, cependant, est l'être, pris comme substance. Ce qui correspondrait à peu près à l'être de la Nature.

Ce n'est qu'à l'époque moderne, et notamment au XVII'me siècle qu'on commence à distinguer la métaphysique comme philosophie de l'être, et la philosophie de la Nature, considérée comme une partie de la philosophie de l'être. À la même époque, avec Kant, la métaphysique devient une philosophie critique, i.e. une philosophie de l'bomme comme sujet critique.

Avec l'époque contemporaine (phénoménologie, et phénoménologie existentielle), l'idée de projet de l'bomme entre définitivement dans la philosophie.

Il y a donc glissement et évolution de l'objet de la métaphysique, quand on la juge encore possible.

Aujourd'hui encore, on considère (cf. GUSDORF) que les thèmes de la métaphysique sont le monde (Nature), l'bomme et Dieu. Et le projet de l'homme est identifié à l'homme lui-même.

Notre approche, au contraire, attribue 6 thèmes à la métaphysique:

1) la situation noétique de l'homme, comme départ de la métaphysique, et qui correspond à peu près à la question «critique ";

2) la Nature ; comme fondement de la temporalité en arrière ;

3) l'bomme, comme fondement de la temporalité au cour de l'immanence, notamment dans la révélation de l'instant;

4) le projet de l'bomme, dans la temporalité, en avant ;

5) la transcendance en personne ; (dont il sera question plus loin);

6) le destin de l'brmme, ou le projet de la transcendance sur l'homme.

Ces six thèmes sont les aspects de l'être-concernant-l'homme. En ce sens, on peut encore dire que l'être est l'objet de la métaphysique, mais non plus l'être abstrait, ou un être vide.

En ce sens, il semble que le discours métaphysique trouve mieux tout son objet, et le discours métaphysique apparaît davantage comme une cuvre humaine, à la mesure de l'homme, en même temps qu'ouvert sur ce qui dépasse l'homme en l'enveloppant.

11. S'il est apparu nécessaire de passer de la question de la situation noétique de l'homme à l'affirmation d'une immanence (c'est-à-dire d'une première totalité 
incluant l'homme et sa situation) (cf. propositions 9, 9.1, 9.2, 9.3), il va maintenant apparaître que l'immanence est finitude, et qu'il faut passer de l'immanence à la transcendance. On va voir que non seulement l'homme a une puissance transcendentale quant à ses objets immédiats, ceux de l'immanence, mais est tenu de reconnaître que lui-même, la Nature et son projet d'homme NE SONT PAS TOUT LE REEL, que quelque chose ou quelqu'un dépasse l'immanence qu'il vient de définir comme temporalité. Comment faire cela? Comment dire cela?

Il suffit de voir deux choses complémentaires: que si la Nature a fait l'homme, elle a la puissance de le faire, mais NE LE SAIT PAS ; puisque dans la Nature, c'est l'bomme qui sait; et que, d'autre part, si l'homme sait, il n'a ni la puissance de se faire lui-même, ni celle de faire la Nature.

Bref, lhomme apparaît ici, à un moment qui est capital et central pour tout le discours métaphysique, comme CELUI QUI SAIT QU'IL SAIT, QUI SAIT QU'IL NE S'EST PAS CAUSÉ ET QU'IL N'A PAS CAUSÉ LA NATURE, ET QUE LA NATURE, QUI, ELLE, L'A CAUSÉ, NE LE SAIT PAS.

On a ici deux puissances et deux impuissances: la Nature peut faire l'homme, et I'homme peut savoir; la Nature ne peut savoir (autrement que par l'homme), et l'homme ne peut se faire lui-même.

Cette proposition complémentaire dit quelque chose que l'on CONSTATE, mais c'est l'homme qui voit que d'elle sort l'évidence de la FINITUDE de l'immanence. Il voit, s'il accepte de le voir, que la Nature et lui ont partie liée pour dire ensemble, chacun à leur manière, quils ne sont pas le PRINCIPE. Le fait que l'homme est séparé, CONNAÎT, transcende la Nature, mais FAIBLE à tous autres égards, éclate devant notre esprit comme vérité centrale.

11.1. En définissant la situation noétique de l'homme, au départ, on voyait bien qu'il n'y a pas de fait primitif évident autre que celui "qu'il n'y a pas d'évidence". Il fallait marcher, continuer, et demander de quoi l'on vit.

On s'est alors reporté sur la Nature, l'homme et le projet. Nous avons appelé "immanence " cette triple réalité solidaire, parce que l'homme s'y trouve inclus. Mais nous ne savions pas encore, à ce moment-là, qu'il y a une transcendance. Au lieu d' "immanence", nous aurions pu alors dire " réalité ", ou « totalité ".

Mais avec une réflexion sur la Nature, qui est toute agitation, toute mobilité inconsciente aboutissant à l'bomme, et avec une réflexion sur l'homme, qui est tout nature (comme dit Pascal), mais aussi tout différent d'elle, comme conscient; et surtout avec cette double réflexion unifiée, apparaît le FAIT RÉFLEXIF PRIMORDIAL, d'où le discours repart, et d'où il ne pouvait pas partir. Au départ il fallait en effet reconnaître l'état de clair-obscur où l'homme, exigeant l'évidence, ne se la voyait pas donnée d'emblée.

Et c'est alors que le mot « immanence " prend un sens. "Immanent " se dit de ce qui est enfermé, inclu, et qui aurait la puissance de ne pas l'être. Ce qui est immanent ici, à la totalité Nature et homme non diversifiée, c'est précisément l'esprit de l'hamme, qui sait se dégager de cette totalité en rejoignant le PRINCIPE (dont l'homme ne peut encore rien dire d'autre que le fait qu’il EST, de toute nécessité). 
Le fait de l'immanence ne prend tout son sens que lorsque l'homme aperçoit celui de la transcendance.

11.2. L'homme apparaît bien ici comme ce qu'il est : le plus faible et le plus fort, et les textes d'Aristote et de Pascal, ou même de Locke (An Essay Concerning Human Understanding, livre IV, chapitre X), s'éclairent mutuellement. Le plus faible, parce qu'il tient son être de toutes choses autres que lui-mêtme; tout l'a précédé et a contribué à le faire apparaître; le plus fort, parce que seul il sait, peut réfléchir sur son être, ET DIRE SA DÉPENDANCE, EN MÊME TEMPS QUE LA DÉPENDANCE DU MONDE, par rapport à ce qu'il appelle alors une cause puissante th intelligente.

Car de rien rien ne snt. Et du non-intelligent, aucune intelligence ne sort. Et du non-vouloir, aucun vouloir ne sort. Mais il faut, pour que l'homme apparaisse : intelligent et voulant, esprit et liberté, une cause qui est au-dessus de toute critique du principe de causalité. (C'est nous qui fabriquons les paradoxes et qui inventons les énormités qui nous permettent de dire sans rire que tout peut venir de rien ou du hasard absolu, ou de la totale indétermination... Comme si le rien, ou le hasard absolu ou la totale indétermination pouvaient être quelque chose... et se déterminer progressivement jusqu’à devenir quelqu'un qui se tient debout sur ces deux jambes et dit : "Qui suis-je ? ").

(Les puissances de négation ont toujours fasciné l'homme, mais davantage encore aujourd'hui, où règne l'imaginaire (il est d'ailleurs bien sympathique, cet imaginaire), où règne surtout le droit de dire tout ce que l'on veut sans avoir à le penser vraiment. Aristote a bien dit qu'autre chose est dire et autre chose penser ce que l'on dit.)

12. Objection: En affirmant ainsi l'existence transcendante de quelque chose ou de quelqu'un qui est puissant et intelligent et qui enveloppe l'immanence, qu'est-ce qui vous dit que vous dites vrai? Qui est-ce qui vous dit que vous dites vrai?

Réponse: Rien ne me le dit, parce que la Nature, qui devrait savoir, ne sait pas ; personne non plus ne me le dit, parce que chaque homme est seul, à cet égard, dans son jugement, étant autant (et n'étant pas plus) que quiconque, et qu'il est précisément celui qui peut dire, s'il le veut bien.

Les actes de la métaphysique sont libres. L'homme peut s'abstenir de les poser. De plus, l'érudition n'y fait pas tout : plusieurs grands esprits ont refusé ou refusent de révéler ce que représente la finitude complémentaire de l'immanence, ou ne semblent pas l'apercevoir. À chacun est remis de juger par soi, avant de s'associer avec ceux qui consentent à une telle évidence. La difficulté psychologique semble d'accepter en même temps une évidence et un mystère: la nécessité de dire et l'incapacité de comprendre parfaitement et objectivement une telle nécessité. Ceci rejoint Aristote à propos de la distinction capitale entre ce qui est évident en soi et évident pour nous.

12.1. Est-ce là antbropomorphisme, projection vaine de l'esprit spéculatif (comme le prétend Kant, qui fait alors de l'acte philosophique ultime un acte moral, pratique)? Non. Car / autre chose est comprendre la transcendance en personne et comprendre comment tout dépend d'elle, / et autre chose en affirmer l'existence et le fait que tout dépend d'elle. // Autre chose est comprendre parfaitement, du dedans, et comprendre en devant affirmer, et à partir de la 
nécessité de cette affirmation. On ne comprend pas positivement ce qui dépasse l'immanence autrement que comme le fait qu'il faut qu'existe en soi quelque réalité qui la dépasse et la cause. Et le "falloir " est encore ici de l'ordre de la connaissance, non d'un ordre moral qui serait distinct de la connaissance. Il s'agit donc d'un acte bumain de connaissance, à la mesure de l'homme (force et faiblesse), qui est alors dit métaphysique.

Ici apparaît mieux le caractère prsitivo-négatif de cette forme de connaissance.

H. SPENCER : "An uncaused deity is just as unconceivable as an uncaused universe ". / Témoignage corroboré par celui de saint Thomas: «Quia nos non scimus de Deo quid est, haec propositio : Deus est, non est nobis per se nota, sed indiget demonstrari per ea qua sunt magis nota quoad nos et minus nota quoad naturam, scilicet per effectus ». (Theol. Ia, II, art. 1).

Noter la différence entre l'agnosticisme définitif de Spencer (qui contient une part de vérité essentielle) et le caractère positivo-négatif, métaphysique, du texte de saint Thomas.

12.2. L'homme (et son rôle alors est bien sûr un devoir éthique, un devoir-dire) affirme $1^{\circ}$ qu'il existe une transcendance absolue, en soi, en personne (qui est alors différente de sa transcendance relative, de son pouvoir de connaissance) ; $2^{\circ}$ que cette transcendance est à sa manière réalité, (c'est-à-dire une nature, insondable, mystérieuse) ; $3^{\circ}$ qu'elle est aussi conscience intelligente, à sa manière, (requise comme cause d'un monde où est apparue l'intelligence de l'homme); et $4^{\circ}$ qu'elle est volonté libre (comme cause d'une cuuvre planifiée, d'un projet aboutissant à l'apparition de l'homme considéré comme liberté et projet). L'effet ne doit nul part dépasser la cause. La détermination précède le possible, et lui ressemble.

Ceci confirmé par l'autorité d'Aristote parlant d'une impossibilité logique de régression infinie de causes; / contesté par Kant jugeant critiquement impossible de remonter du conditionné à l'inconditionné, sous prétexte que nous sommes pris dans le conditionné.

12.3. Dire que l'homme peut et doit révéler cela que ne dit pas elle-même en paroles la transcendance en personne, c'est remarquer la discrétion absolue de cette transcendance, et c'est magnifier l'homme, en faire l'associé de Dieu dans la révélation. Platon, Aristote, les Stöiciens ne jugeaient pas impossible cette révélation humaine. C'est aussi expliciter cette évidence voilée " que l'homme détient en lui quelque chose de l'être, de la lumière et de la bonté qui sont par essence en Dieu ». C'est attester une participation qu'est venue contester la philosophie critique moderne mais que reconnaissait la critique grecque. // L'attitude dite "positiviste", qui ne voudrait rien conserver d'un discours métaphysique positiv()-négatif, est parfaitement légitime en science comme méthode d'investigation du concret, mais n'est pas défendable comme attitude métaphysique. La science a raison de vouloir s'enfermer dans l'immanence, le conditionné, pour l'explorer méthodiquement. Mais elle n'a pas le droit d'être en même temps sciknce et philosiphie, de nier ce qui clépasse sa méthode. C'est pouryuoi aussi il est dangereux de demander à la science de se porter au secours de la philosophie. (C'est là le risque que court la métaphysique soi-ctisant positive de Bergson). 
12.4. Reporter sur la transcendance, par analogie, le triple fait que l'immanence est nature. conscience et projet, c'est dire que Dieu est Principe. Vérité, Bonté. C'est ici qu'apparaît la ressemblance analogique entre la cause et l'effet, entre l'artisan et l'ouvre: l'un et l'autre peuvent être dits être, vérité, bonté. La métaphysique est onto-thénlogie. Dieu et son auvre ont partie liée ; comme, dans l'immanence, la Nature et l'homme ont partie liée.

(Cf. Soljénitzyne, Discours de Stockbolm).

L'Être, le Vrai. le Bien. l'Un. le Beau : étoffe transcendentale de la réalité. Aux trois premiers transcendentaux $l \cdot U n$ ajoute l'idée de l'indivision de toutes choses, prises séparément, et ensemble, dans l'univers ; // le Beau ajoute l'idée de ce que saint Augustin appelle "splendeur du vrai ». Le Beau est ainsi considéré comme la "bonté du vrai ". Ces notions ne sont ici que mentionnées et demandent approfondissement (cf. $3^{\text {ème }}$ partie). Mais elles devaient être mentionnées, au cours d'une approcbe globale, parce qu'elles indiquent déjà l'unité du sens que postule toute métaphysique et comment cette unité est possible dans un discours où la "participation » prendra elle-même tout son sens. Ce qui caractérise proprement l'époque moderne, en philosophie, c'est la mise en veilleuse de lidée de participation appliquée non seulement aux hommes entre eux, mais intégralement: entre Dieu. la Nature et l'bomme. On commence aujourd'hui à redécouvrir la participation entre l'bomme et la Nature. Il reste à renouer avec un discours intégral sur la participation. Le texte de Marcel, et l'ensemble de la philosophie de Jaspers, ainsi qu'une bonne partie de celle de Ricoeur, de Nédoncelle, de Buber, sont axés sur cette idée. Idée qui est elle-même très ancienne (Cf. Phédon de Platon, $4^{\text {ème }}$ argument, Parménide de Platon, panthéisme des Stoïciens, etc.)

13. Il importe d'insister encore ici sur cette implication onto-théologique d'un passage métaphysique de l'immanence à la transcendance en personne. Ce passage, en ce qu'il a cette fois de positif, en ce qu'il dit la ressemblance entre Dieu et son cuvre, entre Dieu et l'homme, éclaire singulièrement le caractère transcendental de l'bomme dans l'immanence, caractère que la philosophie moderne critique a retenu mais ne sait plus comment justifier (Cf. Sartre).

La métaphysique tente en effet de dire que c'est parce que précisément il y a du divin en l'homme que l'homme peut connatitre et est libre, que le monde ò̀ il se découvré est le sien. Au contraire, une bonne part de la philosophie existentielle moderne rôde autour de l'idée de néant. À l'idée de création-participation, elle a substitué l'idée de néant, qu'elle enveloppe d'angoisse ou de révolte. Cette philosophie a souvent mieux permis de comprendre l'homme, sa temporalité, ses aliénations. Il n'est pas cependant requis que de soi elle conteste sans appel l'existence et le rôle actif de la transcendance en personne dans l'être. Un discours métaphysique contemporain voudrait récupérer ce qui, dans la Nature et dans l'homme, fonde toutes les puissances du monde et donne un sens à l'aventure temporelle.

14. Mais alors l'implication du passage de l'immanence à l'absolu, i.e. du fait que les deux ont partie liée, signifie quelque chose d'autre encore: Dieu, avons-nous dit, étant. étant intelligent, et n'agissant dans la création que pour une fin et stlon un projet, le destin de l'homme est précisément d'être une chose voulue, intelligible et bonne. Non seulement l'homme est intelligent et peut connaitre, et agir sur soi et sur le monde, bic et nunc, dans le temps de 
l'immanence, mais la mort, qui semble mettre fin à son projet et à un premier système (immanent) de valeurs, ouvre son destin sur l'au-delà du temps, QUI NE LE SATISFERAIT D'AILLEURS PAS ; elle l'associe à un devenir dont seul le projet de Dieu détient tout à fait le secret. L'immanence (le devoir de vivre) de l'homme ne prend vraiment tout son sens que par la mort. (Cf. le Pbédon). L'homme est effectivement un être-pour-la-mort; / et là encore, une proposition aussi étonnante peut signifier deux choses : que tout le sens de l'homme est avant la mort; ou que tout le sens de l'homme est après la mort. L'époque moderne opte plutôt pour le premier sens. Son pessimisme est ainsi assez notoire. Mais il reste toujours possible d'apercevoir que ce sens, qui est précisément un non-sens, ne s'impose pas. Au contraire, ce qui est absurde c'est d'accepter l'absurde. La vraie révolte est de refuser l'absurde, non de refuser une existence qui en grande partie nous est donnée et dont le sens en grande partie ne dépend pas de nous (comme les Stö̈ciens l'ont si bien vu).

Si le propre de la métaphysique est de "tenir ensemble, dans le sens, ce qui, séparé, en serait dépourvu ", s'il est " volonté de sens ", effort vers le sens, et non déréliction devant la tâche de comprendre, le destin de l'homme est associé à une transcendance vers laquelle il a fallu outrepasser l'immanence, et qui se révèle intelligente et bonne (quoique non moins sévère), à l'instar de la Nature et de l'bomme. Il ne s'agit pas de complimenter Dieu, mais de ne pas lui refuser le sens de la justice dont nous nous croyons à bon droit capables. Il serait en effet étonnant que l'on puisse refuser à la Nature et à Dieu une orientation de justice dont nous nous ferions nous-mêmes un rempart.

Or l'invincible réalité passe par la mort, par notre mort, par la mort de toutes les civilisations (comme disait Valéry).

L'homme a souvent pressenti et affirmé son immortalité personnelle et la sur-valorisation qu'elle signifierait pour lui. Un discours métaphysique (qui cherche moins à prouver qu'à dévoiler et révéler) veut expliciter ce sentiment à titre d'une suite naturelle de ce qui est compris comme déjà fait par Dieu dans la Nature et déjà donné à l'homme. L'immortalité de l'homme ne doit pas être comprise comme une espèce de garantie pour l'avenir, mais comme garantie du sens présent de l'existence. Si l'homme n'est plus rien après la mort, il faudrait qu'il en décide lui-même, ici, tout de suite. Or il n'en peut rien décider, si ce n'est en suivant (comme dit Spinoza) la tendance naturelle selon laquelle l'être est fait, chacun selon son ordre, pour se maintenir. L'immortalité personnelle doit donc être plutôt comprise comme la revendication ultime de l'homme, c'est-à-dire d'un être qui a émergé, sans qu'il le demande, dans le sens d'une existence consciente qui, de soi. demande inconditionnellement de se maintenir. Il faut voir l'immortalité personnelle comme le droit de mourir, de sortir d'une existence imparfaite où est pourtant commencée, dans la parfaite conscience de l'instant, le depassement de l'immanence et de la finitude. Elle n'est pas (comme on le pense souvent, de façon naïve) une espece de récompense ou de condition de récompense de la vie. Et, ici encore, il dépend de l'homme de dire cela que la transcendance en personne lui demande de dire, s'il le veut.

Ainsi, cette fois encore, la totale discrétion de Dieu quant à son projet sur l'bomme n'est pas à déplorer, mais à mettre au compte du fait que l'homme est appelé par Dieu à se définir lui-même jusqu'au bout, malgré le mal apparent et réel, dans la dignité et la dépendance qui lui conviennent. 
15. Le destin de l'homme, $6^{\text {ème }}$ thème de la démarche métaphysique, apparaît ainsi de faģon élargie, et correspond à tout ce qui est advenu, advient, adviendra à l'bomme ; correspond à tout ce que l'homme est appelé à être, dire et faire, de la naissance à la mort et au-delà de la mort : naissance, croissance, éveil de la conscience, connaissance et liberté, science et sagesse, travail, procréation de l'espece, action sociale, souffrance, joies, vieillissement, attente du mystère, déclin, départ.

Ce thème est donc plus large que celui du "problème du mal ", auquel on l'identifie souvent. Il est celui de lacomplissement de l'bomme, de tout le bonbeur de l'homme, dans le temps, dans l'au-delà du temps déjà commencé dans l'au-delà de la vie humaine et de l'histoire. Il relève d'un triple projet : le projet de Dieu sur la Nature et sur l'homme, le projet de la Nature (l'apparition de l'homme), le projet de l'homme compris dans toute l'extension de ce qu'implique pour lui un projet.

15. I L'homme, par son destin, est mis en double situation temporelle et intemporelle, et le fime thème rejoint le premier, qui au départ était celui d'un étonnement d'être, d'une difficulté de savoir, et qu'on a appelé imparfaitement "situation noétique ».

D'où, comme il était à prévoir, la « circularité " de la démarche métaphysique, circularité qui lui est essentielle, non pas à titre de prétention vaniteuse, mais de "discours humain sur le sens de la totalité ". Comme être de vérité, l'homme ne peut se satisfaire de rien de moins que d'une certaine comprébension de la totalité. Le scepticisme ne porte pas sur la totalité ; il n'a tout simplement pas d'objet; il s'abstient. Un discours humain métaphysique ne prétend pas comprendre tout, mais quelque chose du tout.

15.2 Ce discours n'est done "dogmatique " (si on veut retenir le mot) que si l'on entend par là une volonté invincible et inaliénable de briser la totale indétermination et le pur étonnement à quoi pourrait aboutir un discours qui se voudrait philosophique.

Mais il faudrait plutôt dire que le discours métaphysique est essentiellement ouvert, par ses six thèmes et par chacun d'eux, à un mystère qui ne sera jamais, ici et maintenant, parfaitement éclairci, parfaitement déployé.

Ce mystère est de tous les instants, à tous les instants, mais aussi de l'histoire, c'est-à-dire d'une vie à plusieurs. Non seulement il est insondable dans l'instant, mais il est imprévisible dans l'histoire. Il est riche de tout ce qui dépasse l'homme, au-delà de l'immanence, mais aussi de tous les développements imprévisibles de l'histoire, dans l'immanence.

Et pourtant, une certaine métaphysique est "faisable", parce que la race humaine est entière en tout homme, qu'il est donné à tout bomme, à l'homme quotidien, de se comprendre dans la verité. L'homme abstrait n'existe pas, pas plus qu'une humanité abstraite ou que des faits bruts. Ce qui existe, ce sont des personnes humaines, tout aussi valables les unes que les autres. Il n'y a pas d'esclave métaphysique. Tout bomme est métaphysique, sinon dans le discours savant et habile, du moins dans l'esprit. Tout bomme détient l'essentiel de ce qu'il doit savoir. Son être se suffit.

Locke a admirablement écrit sur cette " condition suffisante" de l'homme. Il fait bien voir que nous sommes essentiellement "voyageurs» (Homo viator, 
repris par Marcel), que nous avons tous les moyens du bord pour naviguer dans un monde où une bonne part de la viabilité, du bien-être, dépend de chacun et de tous.

15.3 De même, autre chose est dire qu'il y a de l'absurde dans le monde, autre chose que le monde est absurde. En fait, la lumière (une lumière suffisante) y voisine avec l'obscurité, le plaisir avec la peine, le déterminisme avec la liberté, le mouvement avec l'attente, l'expérience avec la désinvolture, la naissance avec la mort, le temps avec l'éternité commencée.

Si l'on hésite parfois à dire avec trop d'assurance que ce monde a un sens, ce n'est pas parce qu'il n'y a rien de clair ou que le sens est irrémédiablement mal indiqué, ni parce que nous n'aurions comme seul recours que la critique, mais parce qu'il y a effectivement des frères qui souffrent, qu'il est facile d'être téméraire en métaphysique, et qu'en affirmant ce que l'on croit péremptoire, on ne doit jamais se permettre de se substituer à l'intelligence et à la liberté d'autrui ; c'est surtout (on l'aura souvent dit), que le mystère demeure, au-delà de tout discours.

\section{L'objet de la métaphysique}

L'homme, en étant et en le disant radicalement, c'est-à-dire en le disant comme sens, révèle autant qu'il est. Par lui le sens métaphysique (i.e. du tout et du "pourquoi quelque chose ") entre dans le monde. L'homme est le tout de ce qui est, mais pour autant que le vrai est ce qui est et que le vrai passe par lui, est soumis temporellement à son vouloir-dire. Tout, de cette vérité qui ne peut se dire elle-même, est soumis au discours de l'homme, bormis le cas d'une révélation divine extraordinaire, ce qui est exclu comme tel en philosophie. Seuls révèlent ici la Nature et l'homme, et encore, la Nature par l'homme. Dieu, comme tel, est absent. Le discours métaphysique (comme d'ailleurs tout discours) est lié au fait humain, mais d'une façon qui fait que l'homme n'est pas à proprement parler l'objet de la métaphysique. Comme le dit bien Marcel, l'homme participe à cet objet autant et plus qu'il ne l'objective. C'est donc en un sens étroit qu'on pourrait dire que l'homme est l'objet du discours métaphysique.

On peut dire, prudemment, que c'est l'être qui demeure cet objet : l'être des six thèmes ici énoncés: car une situation humaine est de l'être pour l'homme; de même, la Nature est bien sûr de l'être; et l'homme, et son projet, et Dieu (être par excellence), et aussi est de l'être ce qui advient de l'homme. Mais non plus seulement une notion "logique " d'être. L'être, au contraire, qui dit d'abord relation réelle à l'existence.

De plus, il faut bien dire que la notion de "fait " a brouillé à cet égard bien des discours. Qu'est-ce qu'un fait? ce serait l'être sans son mystère. Il n'y a pas de tels "faits bruts", sauf par mise entre parenthèses méthodique, pour fins scientifiques. Le fait est vérifiable, l'être est vérité. Et alors il est inséparable de la liberté et de la création: liberté et création bumaines, et (plus profondément), liberté et création divines.

16. 1 On accède ici à quelque chose qui aide à comprendre ce que l'on pourrait appeler, avec réserve, la "misère " de la métaphysique. Les trois types de connaissance définis à la proposition 5 ont tous quelque chose d'imparfait ; ils sont autant d'aspects d'une activité humaine qui ne peut saisir d'emblée le 
réel : la connaissance perceptuelle accorde notre corps, nos sens, notre sensibilité, avec ce monde qui se présente à nous d'abord comme notre "seul bien »; la connaissance scientifique, elle, cerne de beaucoup plus près cette concrétude phénoménale, mais elle laisse tomber tout le pittoresque du monde (seul en effet le scientifique lui-mêtme trouve pittoresque son appareil scientifique de connaissance); la connaissance métaphysique fait pénétrer l'homme dans les rapports subtils, existentiels, que tous les êtres ont entre eux, de l'bomme, par la Nature. jusquià Dieu et au lien qui unit l'bomme à Dieu, mais il a fallu pour cela renoncer au pittoresque de la vie sensible et aux assurances intellectuelles de la science.

Il faudrait une quatrième sorte de connaissance, qui serait davantage celle de tout l'bomme, une fois qu'il a longuement fait l'expérience des autres connaissances, (pour autant que cela est possible), et qui se rapprocherait alors davantage de la connaissance globale, sensible, du premier type. Ce serait alors celle où, sans être exclusivement sensible, ou abstraite comme la science, ou prisonnière du discours comme la métaphysique, toute la personne humaine se sentirait en harmonie avec un objet, le réel, qu'aucun artifice ne séparerait plus d'elle, de sa vie, vécue comme une totalité concrète.

Cette connaissance toute personnalisée, à la fois sensible, intellectuelle, morale, est sans doute ce qui se tisse en chacun de nous, au fil des ans et de la multitude de nos expériences, externes et internes. Elle est liée à une certaine affectivité, à un certain respect de la pure nécessité du monde, à la liberté métaphysique de l'âme, mais elle est surtout elle-même. totalement indicible, et en quelque manière religituse, mystique, au sens à la fois laïc et théologique qu'il faut donner à ces mots.

Plus souvent qu'autrement, c'est cette connaissance du $4^{\mathrm{èm}}$ genre qui parle à travers les trois autres, qui motive notre recherche, soutient nos doutes, colore nos intonations. C'est une espèce de foi, plus solide que toute autre espèce de connaissance, et qu'on peut alors chercher à percevoir en autrui, quand on l'écoute. Elle serait la samme de nos expériences, ce que nous pensons vraiment en nous-mêtmes.

La connaissance métaphysique n'a donc pas les vertus de celle-là ; elle est une structure méthodique particulière où l'on choisit de couler une certaine compréhension intellectuelle du réel. Tout discours philosophique, à plus ou moins brève échéance, se réfere ainsi à un schéma plus ou moins avoué et explicite, et qui relève d'études, d'expériences, et d'un effort plus ou moins explicite de syntbiese.

17. Tel qu'on a (par choix) voulu le présenter ici, le discours métaphysique est le projet noétique libre d'un être parmi les êtres, qui connaît sa limitation et sa transcendance relative : c'est-à-dire sa dépendance (il n'a pas fait le monde, qui l'a fait), et son autonomie (il sait que le monde l'a fait et que lui, le monde, ne le sait pas ; il peut et doit aménager un monde qui ne peut parfaitement s'aménager sans lui). Par lui, par ce discours, l'homme se connait mieux (selon le voeu antique), s'assume dans le temps et au-delà du temps, et se remet librement à ce qui le dépasse et peut l'achever.

18. La métaphysique fait usage, dans la présente démarche, de six thèmes-outils. Ces thèmes sont apparus comme liés entre eux, au cours d'une exploration sur le sens de la totalité. Ils seraient alors ses fondements, pour autant qu'ils 
recouvrent distinctement et adéquatement l'ensemble du réel et pour autant que, sans l'épuiser, ils l'expliquent.

19. À l'intérieur de ces six thèmes est apparue une quadruple complexité, selon que le départ est difficile, que le réel de l'immanence est composé et multiple, selon que l'homme doit dépasser l'immanence pour le comprendre, et selon que le projet de la transcendance reste toujours lointain pour qui cherche à le comprendre du fond de l'immanence:

complexité : 1) de situation de l'homme (au départ);

2) de composition;

3) de dépassement ;

4) d'intention, de gratuité, d'éloignement.

Comprendre, "tenir ensemble métaphysiquement ", au delà de la connaissance perceptuelle et scientifique, c'est avoir d'abord reconnu et connattre chacun de ces thèmes, selon son sens, sa mesure et son mode, et les tenir ensemble, sans rien y sacrifier, d'une quadruple et unique manière correspondant aux quatre modes de la complexité métapbysique du réel.

20. Cette compréhension apparaîtra de mieux en mieux comme une ontologie (par où un discours métaphysique est possible), une théologie (par où ce discours est ultimement fondé), une phénoménologie (par où il est humain, temporel, et se répand dans toutes les apparences), pratique (par où il ne veut éluder aucun des devoirs de l'homme).

Entreprise définie par son universalité même (et non par sa spécialité), et qui par là veut n'être étrangère à rien, n'être liée à aucun parti.

21. Cette compréhension est ordinairement pressentie et variablement vécue par l'bomme quotidien. Elle s'est exprimée, à des degrés divers, et parfois dans l'agnosticisme ou le refus, selon les époques de l'histoire de la pensée et de la culture.

21. 1. Comprendre métaphysiquement, c'est donc aussi connaître et reconnaître ces six thèmes selon une pondération bistorique qui est en même temps une «interprétation".

À travers l'homme historique, en effet, tous les sens de l'être vont et viennent, apparaissent ou parfois s'estompent, ou s'explicitent. Il y a là une manifestation particulière de l'bomme, dans sa pensée et sa liberté, dans son adaptation au temps et aux circonstances, pour autant qu'il est lié à certaines déterminations bistoriques et à une communauté. Les multiples possibilités de la Nature et de l'homme ne sont jamais toutes données explicitement à un moment de l'histoire, mais étalées sur le temps. Non seulement l'homme-individu doit se connattre, mais aussi l'homme-universel (qu'il ne faut pas confondre avec l'homme abstrait).

Bien des difficultés du discours métaphysique viennent de ce que l'on oublie cela, et qu'on est prisonnier d'une manière unique de voir les choses, liée au moment présent. Le discours métaphysique, par essence, veut libérer. Il se veut sagesse, non discours spécialisé sur tels ou tels objets. Mais en même temps capable de relier entre eux tous les objets, toutes les aspirations de l'homme. À cet égard, le projet de l'bomme, $4^{\text {ème }}$ thème, est un bon exemple de thème qui est apparu assez tard dans l'histoire, avec toutes ses implications, et qui, 
aujourd'hui, a tendance pour cela à se trop maintenir dans l'immanence. Seule une interprétation métaphysique de l'bistoire peut rectifier ces excès inévitables, en pondérant les rapports des aspects de l'être, selon les circonstances.

21.2 Certains hommes, artistes, politiques, penseurs, visités davantage pat le génie, ont conceptualisé ou autrement exprimé, ou réalisé, certains des sens particuliers de lêtre. Et particulièrement, en ce qui nous concerne ici, les Philosophes. Ainsi : Platon, le discours; Aristote, la substance; Plotin, la procession des êtres à partir de l'Un et leur retour à l'Un; $S$. Augustin, l'expérience de la solitude et de la déréliction de l'homme, sans Dieu; $S$. Thomas, le rapport métaphysique de l'essence et de l'existence et le rapport de l'homme à Dieu; Bacon, la tâche technique de l'homme ; Descartes, le rôle déterminant du moi humain ; Locke, l'équilibre naturel des choses ; Berkeley, la transparence du sensible ; Hume, le doute, inhérent à toute connaissance humaine ; Kant, le fait de structures noétiques et morales, hiérarchisées en l'homme et lui permettant d'être connaissant et agissant dans le monde ; Hegel, les implications du devenir et de l'histoire, l'historicité même de l'homme; Marx: la priorité relative du concret; Bergson, les nouveaux rapports de la psychologie et de la métaphysique, la créativité ; les existentialistes : le rôle du néant dans la connaissance de l'être ; etc., etc.

C'est cette idée que Karl Jaspers évoque ici : "L'ensemble de la tradition nous a laissé un résidu précieux de vérités inépuisables et nous montre les voies nous permettant de philosopher aujourd'hui. La tradition, c'est la profondeur apergue, avec une attente inlassable, de la vérité qui s'est faite pensée ; c'est la richesse inépuisable de quelques grandes auvres ; c'est la présence de grands penseurs, accueillie avec respect ". (Introd. à la philosopbie, traduction J, Hersch, 1962, p. 198.).

21.3 Cette attente inlassable n'élimine en rien la tâche bumaine à faire ("praxis", comme disait déjà les Grecs, et comme le redit autrement Marx). Marx (après Hegel et Feuerbach) a justement dénoncé les ALIÉNATIONS qui ENCOMBRENT inévitablement la marche bumaine et s'établissent solidement en STRUCTURES aliénantes: métaphysique abusive, économie abusive, état abusif, religion abusive, etc. Il a cru devoir dire qu'il faut cesser d'interpréter métaphysiquement le monde et qu'il ne faut que le CHANGER. (CF. onzième thèse sur Feuerbach). De même Nietzsche refuse une métaphysique qui se donnerait pour accomplie définitivement sur des essences en soi, et proclame le sens unique de la vie et de l'avenir. Ces deux témoignages philosophiques ont leur valeur, mais le dernier mot sur tout ne s'y trouve pas dit pour autant.

Il faudra toujours interpréter le monde, selon le passé, le présent, la Nature, les acquis, les déficits, selon ce qui reste à faire, et selon ce qui manque à l'homme, dans l'immédiat, pour se bien comprendre.

Socrate dirait sans doute aujourd'hui que toute idée peut devenir aliénante, aussi bien celles dềtre logique, de Nature, de Dieu, de Liberté, de plaisir, de projet, de société, d'inconscient, d'aliénation . . . L'homme n'est jamais tout à fait ce qu'il a à être. La connaissance doit le libérer, libérer en lui l'être.

22. Puisque la métaphysique est un projet noétique à plusieurs, l'outil (la structure) qu'on propose ici demande d'une part d'être confronté à l'histoire de la pensée philosophique, pour qu'il apparaisse légitime d'en faire usage au cours d'une ANALYSE plus approfondie des aspects de l'être, objet total de la 
métaphysique. D'autre part, on sait maintenant d'avance que ce projet restera toujours imparfait, inachevé, à cause du mystère lui-même, de l'amplitude du temps, et des différences individuelles. L'homme passe par "un milieu des choses ". Il vit, observe, interprète, agit. Il est médian et médiateur, et l'effort est sa loi. Il participe au monde, aux autres, à Dieu; il leur est présent, en même temps qu'étranger. Il est et devient. (Pindare: "Deviens ce que tu es $\gg$.

23.

Vent dOuest ${ }^{1}$

Quand ce vent s'est levé qui m'ordonne de vivre,

Surgi du plus obscur que ma raison prétend ; Quand, l'effort du gamin tirant sur l'occident Le cerf pur, affolé, que notre plage enivre,

La machine a cherché l'azur qui la délivre; Alors m'est apparu qu'un séparable étant, Qui n'a qu'on n'apprivoise et qui serait néant,

M'incite à l'employer plutôt que de le suivre.

Le Principe est à l'est, où tremblent nos appuis,

Et c'est en l'accident que l'âme se construit : Il lui faut des objets, un levier, l'air tragique.

Je m'élèverait donc, contre l'air, sur midi, Sans clamer que je sache ou doute d'où je suis :

Ma voie est mitoyenne, et moi, métaphysique.

\section{PONDÉRATION HISTORIQUE DES SIX THÈMES}

24. Il y a quelque fondement à une division quadripartite de I'histoire du développement philosophique en Occident, soit :

1) l'antiquité ; pensée philosophique, originelle, multiple, indissociée ;

2) le moyen agge (300-1440 A.D.) : pensée onto-thénlogique, protégée, sotériologique ; associée ;

3) la modernité (1440-1800); pensée épistémologique, vérificatrice

(Cf. Koyré : Du monde clos à l'univers infini) ;

Bacon-Galilée : les faits bruts et les lois ;

1. Pour avoir observé, sur une plage américaine, comment un gamin se trouvait faire nier la pesanteur à son cerf-volant en le tirant contre une brise venue de la terre ferme. "La terre nous en apprend plus long sur nous que tous les livres. Parce qu'elle nous résiste. L'homme se découvre quand il se mesure avec l'obstacle. Mais pour l'atteindre, il lui faut un outil (..), et la vérité qu'il dégage est universelle ". (Saint-Exupéry, Terre des Hommes). 
Newton-Locke : avènement des « lumières "; ambiguité des rapports foi, métaphysique et science; pensée dissociée: rançon de la critique; transition vers des valeurs nouvelles;

4) l'époque contemporaine : pensée phénoménologique, parallèle à la métaphysique ; exaspération de la liberté et de l'humanisme; recherche soupçonneuse de l'humain ; bienfaits et dégâts de la technologie ; critique des pouvoirs et de l'inauthentique ; autonomie de l'art et de l'imaginaire ; découverte de l'interdisciplinaire ; nouveaux dialogues ; redécouverte de l'être ; besoin de simplifications ; nouvelle aspiration, épurée, d'une discipline logique, morale, politique et religieuse; valeur irremplaçable du pluralisme et retour aux sources, en même temps que fuite en avant, et prospective.

Grille de pondération historique des thèmes

$$
\text { Ant. (1) Med. (2) Mod. (3) Cont. (4) }
$$

a)

$\begin{array}{cccc}\text { Ant. (1) } & \text { Med. (2) } & \text { Mod. (3) } & \text { Cont. (4) } \\ \mathrm{x} & \mathrm{x} & \mathrm{x} & \mathrm{x} \\ & & & \\ \mathrm{x} & \mathrm{x} & \mathrm{x} & \mathrm{x} \\ \mathrm{x} & \mathrm{x} & \mathrm{x} & \mathrm{x} \\ \mathrm{x} & \mathrm{x} & \mathrm{x} & \mathrm{x} \\ & & & \\ \mathrm{x} & \mathrm{x} & \mathrm{x} & \mathrm{x} \\ \mathrm{x} & \mathrm{x} & \mathrm{x} & \mathrm{x}\end{array}$

f)

b)

c)

d)

e)

- «Rien de réel n'est qu'une seule chose " (Valéry, Cabiers, IV, 836).

- Présence, oubli et récurrence de la vérité.

- Difficile accord de la vérité et de la vérification.

- Démystification de la conscience claire, des "isme" ou idéologies pathétiques.

- Prix onéreux souvent payé à la polémique: du temps ordinairement perdu.

- L'humanité évolutive : la même et changeante. Croire aux deux, voilà le difficile, le nécessaire, le métaphysique.

- Avertissement : caractère imparfait, exploratoire, de cette pondération.

26. (a-1). - Pensée ingénue, indissociée, non idéologique, amie-de-la-sagesse, philo-sophique. - La pensée comme étonnement essentiel, comme "embarras", i.e. "aporie "; la pensée comme dévoilement (alètheïa) ; la pensée comme équation sujet-objet (Parménide); la pensée comme mesure (Protagoras ... Platon...); la pensée comme mesure de l'action (Socrate); la pensée comme outil (les sophistes); le jugement suspendu (épochè) (Épicure, Pyrrhon), etc. ; la pensée "logique ".

27. (a-2). - Pensée protégée et participée, théo-logique ; "croire pour mieux comprendre ", "fides quaerens intellectum "; Dieu-vérité ; débat des "deux " vérités. Retrouvailles de l'esprit avec luimême ..., rendant possible l'argument dit ontologique. Réunion de l'esprit et de l'élan affectif; sécurité noétique inégalable, quoique séparée des modes concrets du savoir (les sciences concrètes viendront plus tard). 
28. (a-3). - Pensée épistémo-logique (par opposition à pensée sapientielle), (v.g. Galilée, Descartes, Bacon). L'aphorisme de Bacon indique la direction générale: "Natura parendo vincitur » : "Nature to be commanded must be obeyed". Ceci rejoint le propos de Heidegger sur ce qu'est la science (au début de Qu'est-ce que la Métaphysique ?) - Naissance du Mécanisme moderne (Cf. Mersenne 1588-1648). - Pensée critique : Cf. Locke, Hume, Kant ; débuts de la pensée historique (Vico 1668-1744). Avènement des Lumières, i.e. du "rationalisme "; le siècle des "philosophes "; - Pensée ensuite dite positive (Comte 1798-1857), Spencer (1820-1903); vers le positivisme logique, au siècle suivant.

29. (a-4). - Pensée phénoméno-logique, et toutes ses implications; (Hegel, Dilthey (1833-1911), Nietzsche, Husserl, Bergson (1859-1941)); pensée sociologique (Comte) (Durkheim, le structuralisme à venir); pensée dialectique, nourrie de contradictions et de médiations (v.g. Marx); sciences de l'homme; prolifération illimitée des sciences ... ; morcellement du savoir ; pluralisme des « spécialités». Révolte et non-savoir.

30. (b-1). - Prééminence du cosmos, du cosmo-logique; i.e. du sens du mouvement dans l'ordre, des corps célestes; prééminence de la nature (omne vivens ex vivo); philosophie de la génération et de la corruption ; caractère irréfragable de la nature, alors apparentée au divin et au sacré.

31. (b-2). - Maintien de la nature comme source première, non connaissable, mais doublée de la surnature, la grâce (gratuite. . ), le surnaturel. Dans l'antiquité on disait que l'art imite la nature; ici on dit que la grâce ne détruit pas la nature.

32. (b-3). - Observables " en soi ", obéissant à des "lois " concrètes, le cosmos et la nature sont progressivement détachés de la transcendance en soi (le sacré, Dieu) en faveur du "transcendental ", via l'entendement humain, et apparaissent de plus en plus comme "positifs": c'est-à-dire comme n'évoquant pas de réalité-inconnaissable (i.e. négative).

33. (b-4). - L'idée de " développement " ou « évolution " à l'intérieur même des "espèces". - Donc, débordement du "devenir "sur les "faits"...; relativité de plus en plus avouée des faits... (Einstein, Heisenberg) ; l'homme comme produit de la nature et débordant la nature...; redécouverte de la nature (écologie, etc.).

34. (c-1). - Découvertc incomplète de l'homme (v.g. les sophistes; Socrate : "Connais-toi toi-même»). - Identité de l'homme et de l'âtne, v.g. chez Platon; l'homme tragique: Cf. Eschyle, Sophocle; l'homme comique: Cf. Aristophane; l'homme éthique et politique ; les dicux-surhumains ; ségrégation homme-libre et hommeesclave.

35. (c-2). - L'homme sauvé, sur-élevé ; image de Dieu. - Valorisation incontestable de la "personne " humaine. Dieu parmi les hom- 
mes : l'Homme-Dieu. - Promesse d'une résurrection de la chair. - La nouvelle communauté humaine: l'Église. - Effacement relatif de l'homme mondain, civique. Apparition des nationalismes, d'un type d'homme qu'on dira plus tard " moderne".

36. (c-3). - Renaissance de l'bumanisme : Cf. Dante, Pétrarque, Rabelais, Léonard, Montaigne. - Réformes . . ., révolutions . . . ; recherche d'un idéal démocratique (liberté, égalité, fraternité).

37. (c-4). - Le " phénomène humain " reconnu (Cf. Teilhard); prolifération des manifestations de l'humain. - Terre des hommes. Intersubjectivité mieux comprise ; l'idée $\mathrm{d}$ "apprivoisement"; $l$ "acceptation inconditionnelle d'autrui"; proliferation des communications ; Tiers-monde ; décolonisation, intégration, etc. ; droits de la femme et de l'enfant ; responsabilité collective ; etc.

38. (d-1). - Projet limité, éthico-politique; non-technologique.

39. (d-2). - L'idée de "salut éternel " domine le projet, au-delà. Cependant, l'humanité "en marche " apparaît aussi davantage que dans l'antiquité ; de Rome et d'Athènes, la chrétienté a retenu l'idée d'un projet politique maintenant fondu dans l'immense brassage social que représente le moyen âge.

40. ( $₫-3$ ). - Grandes explorations dans tous les domaines; découvertes techniques de plus en plus nombreuses et préparation du grand âge technologique. - L'idée de progrès se dégage progressivement. Utopies scientifiques, sociales et politiques.

41. (d-4). - Passage de la civilisation industrielle à la civilisation post-industrielle après le " climax " coïncidant avec le tournant du siècle. Les premiers projets internationaux (S.D.N. et O.N.U., etc.). Progrès des philosophies sociales. - Progrès inoui des communications, rendant possible le projet total de l'humanité. Redécouverte des limites de l'homme; appel aux arrêts de croissance (Le Club de Rome) ; - Redécouverte du sacré comme dépassement possible du projet humain.

42. (e-1). - Crainte révérentielle et affirmation rationnelle mitigée de l'existence de la transcendance en personne: dieu, les dieux. Dieu, $1^{\text {er }}$ moteur et providence. Des pré-socratiques aux stoïciens.

43. (e-2). - Prééminence de la transcendance. Tout passe par ce pôle, (v.g. art et science).

44. (e-3), - L'ilée de Dieu, toujours maintenue, devient équivoque et fait apparaitre le déisme, où Dieu est conçu de plus en plus exclusivement comme une " chose". D'où un mouvement vers l'agnosticisme ou même le rejet total (athéisme) de Dieu et finalement la " mort " de Dieu.

45. (e-4). - Silence de Dicu; recherche de Dieu. "Goilless theology ". Godot. 
46. (f-1). - "Fatum " antique (fatalité) ; mélange d'optimisme et de résignation. Importance de la vertu de courage dans l'antiquité. L'émergence du stoïcisme. L'existentialisme d'Épicure et de Lucrèce.

47. (f-2). - Sublimation du mal, attribué à une faute historique originelle. Le concept chrétien de prédestination, distinct de la fatalité antique, quoique distortionné dans les querelles théologiques sur la grâce et la liberté (Cf. S. Augustin et Pélage 360-440), (Cf. Molina 1535-1600, Calvin 1509-1564 et Jansénius 1585-1638). (transition avec l'époque suivante...).

48. (f-3). - Ambiguité du « meilleur des mondes "; Leibniz (1646-17 16) (théodicée) et Fontenelle (1657-1757 sic); Candide, de Voltaire (1694-1778) ; Bernardin de Saint-Pierre (1737-1814) : Études de la nature ; déroute progressive des causes finales . . ; littérature du " mal "; (transition avec l'époque suivante ...)

49. (f-4). - Philosophie de la révolte et de l'existence. - Philosophie de l'ambiguïté. - Libérations psychologiques. - Les poids de la liberté. Responsabilité et ouverture.

50. Nouvelle indication bibliographique.

51. Apparition, maintien, effacement, intensification ou récurrence historiques des six thèmes. Les Weltanschauungen comme tempéraments historiques. "L'unité de l'expérience philosophique "(Gilson).

\section{Transition}

52. Dialectique du trap et du trop peu (Pascal etc.) appliquée aux six thèmes. Petite histoire des « isme».

Thème (a) - De notre puissance de connaitre (entendement, raison), on peut dire trop (rationalisme) et trop peu (agnosticisme, scepticisme); on peut l'exalter outre mesure, en la disant capable de RÉDUIRE le réel, et le mystère qu'il comporte ; on peut aussi exagérément l'abaisser.

Les deux attitudes s'expliquent aisément

1) par l'urgence pratique et sociale, où nous sommes plongés, de juger, de conclure, de décider, de déclarer, de faire ;

2) et, d'autre part, par la conscience intime que nous avons de n'être pas les maitres de la vérité, de flotter souvent à la surface des choses, d'être les jouets des événements, de déterminismes, etc.

A. Rationalisme. Il est aisément explicable comme tendance. Pourquoi en effet cette puissance (la raison), sinon pour vaincre, surmonter, réduire?

1. La plupart de nos jugements (jugements de faits) sont faciles à faire, portant sur des choses anodines, et peuvent s'autoriser d'être catégoriques : aller, venir, acheter, vendre, visiter, voir, sentir, arranger, organiser, etc. Le fait brut, physique ou historique, s'impose aisément à un usage quotidien du réel, impose spontanément de tirer tout au clair. 
2. Le langage rę̧u donne facilement l'illusion de FIXER la vérité. À la limite, le dictionnaire contiendrait la vérité . . L'imprimé, le médium, alors s'emparent de ces soi-disant vérités, déposées dans les mots, et surmultiplient l'illusion.

3. La logique et les mathématiques donnent aussi le change; se donnent spontanément comme "l'idéal de connaissance vraie". Ici, pour cause, la preuve triomphe. Et pourtant, elle n'est partout que "formelle ", celle d'un contenant sans contenu.

4. Le législatif use de formules dites "légales", univoques, et favorisant encore l'impression que tout est régissable, simple, clair.

5. La science et la technique exigent, pour cause, la précision. Elles se doivent forcément d'escamoter le mystère des êtres, de la vie, des désirs, des personnes, de la profonde complexité sociale. Il faut qu'une porte soit ouverte ou fermée; qu'on sache à quoi s'en tenir sur les poids, les mesures, l'heure des trains, la résistance des matériaux, etc.

6. Même lart finit par s'enliser, être populaire ; il se mue en "formules» et devient "académique". Pourtant «l'ennui naquit de l'uniformité ", et l'art devrait être une perpétuelle création de nouveautés.

7. La religion elle-même devient "établie ", codifie ses rites, ses règles, ses " dogmes". Elle devrait pourtant toujours tout remettre en question, et porter essentiellement sur le mystère.

8. Enfin, les simplifications idéologiques, les étiquettes politiques, ont tendance à faire passer pour défini ce qui reste toujours à définir, à adapter, à transformer.

La philosopbie pourra donc aussi, à son tour, comme reflet culturel, oublier la diversité de l'être ainsi fixée de mille manières. Elle aimera chercher à son tour des FOR MULES, simplifier.

Mais le vrai sens de la puissance rationnelle de l'homme est d'ordonner nos multiples impressions de connaissance, sans les détruire ; sa fonction est de classer (v.g. vers une "cohérence maximale ») : de réduire ce qui est par trop affectif, mais non le réel lui-même, en vue d'une «connaissance la plus objective possible ». Exactitude n'est pas toujours RIGUEUR. La philosophie doit être partout rigoureuse, et exaite là où elle le peut (Cf. Aristote, Métaphysique, livre 2). La clarté trouvera son compte dans ce partage.

B. Agnosticisme, scepticisme.

De même que la raison, comme puissance réductrice, est faite pour rassurer notre pensée changeante et parfois inquiète, et soutenir par là notre "intelligence ", non la remplacer, -

De même, le DOUTE est cette part de notre connaissance qui nous avertit qu'elle n'est jamais parfaite, qu'elle n'épuise rien (Cf. Socrate). Le doute, s'il est sincère, ne dit pas que notre esprit ne saisit rien, mais qu'il ne saisit parfaitement le tout de Rien.

Pourquoi alors oublie-t-on souvent cette vraie signification du doute, et sa fécondité? 
1. parce que la vie problématique de l'intelligence est difficile à porter ;

2. parce que nos sens, notre inconscient, notre raison, les discours, souvent nous ont trompés;

3. parce qu'on se donne parfois l'air d'être sage en doutant de tout; en ne faisant valoir, par ressentiment, que cette virtualité paresseuse et insolente.

\section{Conclusion.}

Si l'homme est capable de dnute et de rigueur, c'est qu'il est appelé à juger, au milien des choses où il se trouve, c'est-à-dire au cœur de ce mélange : clarté-ténèbres, déterminismes-liberté, tradirion-progrès, acquis et tâches à faire, - qui est la vie.

Thème (b) : Nature. -

1) Dire tmp de la nature (i.e. de la matière et de ce qui en surgit), c'est dire qu'elle existe en soi, comme une sorte d'étoffe (anglais: stuff; allemand: Stoff); comme FAIT tout-puissant, capable par soi de tout, et construisant tout mécaniquement, sous couvert de pure complexité, de pur basard, de pure probabilités; comme Fait brut (and so what?) qu'elle semble être ; c'est oublier qu'elle n'est que du TRAVAIL, de l'instrumental.

2) Dire trop peu d'elle, c'est sous-estimer ce qu'elle porte en elle de possibilités réelles (et non seulement statistiques), de projets (donc d'Esprit); c'est sous-estimer la causalité efficiente qu'elle représente, la finalité qu'elle recèle.

Thème (c) : l'bomme, la conscience. - -

1) Dire trop de l'homme, c'est oublier de quoi il est fait et dépend, en arrière.

2) Dire trop peu de l'homme, c'est oublier de quoi il est capable, comme héritier et maître de la Création ; c'est, entre autres choses, oublier ce qu'il est capable de dire, métaphysiquement, et que rien ni personne ne peuvent dire, sans lui.

Thème (d) : projet de l'bomme. -

1) Dire trop du projet de l'homme, c'est la démesure même ; c'est croire exagérément au progrès, s'en remettre béatement à la science et à la technologie, à l'utopie pure ; c'est s'imaginer pouvoir "sortir de la nature ", défier absolument la mort.

2) Dire trop peu du projet de l'homme, c'est se résigner passivement et facilement, s'en remettre à l'effort d'autrui ou à la critique oisive ; c'est oublier la grande espérance qui nous est indiquée dans la nature (v.g. pouvoir rapide de cicatrisation des blessures et des plaies, courage des animaux); c'est oublier les possibilités constructives et adaptives de la jeunesse, la valeur de l'auto-critique personnelle, sociologique et religieuse ; c'est démissionner devant l'effort et les renouvellements possibles; c'est manquer de sens politique.

Thème (e) : là Transcendance. Dieu. -

1) Dire trop de Dieu, c'est prétendre qu'on le connaît quand on a compris qu'il faut qu'il existe ; c'est croire " absolument " aux modes humains de discours que nous formons pour parler de lui et scruter Sa sagesse, Ses projets. 
2) Dire trop peu de Dieu, c'est oublier qu'il dépend de nous qu'il soit dit qu'Il existe; c'est faire paresseusement confiance à des MOTS : matière-en-soi, hasard, progrès, pour fonder toutes choses ; c'est croire que Dieu ne peut pas faire que quelque chose soit, hors de Lui (si l'on peut dire), et venu de rien d'autre que Sa puissance; c'est croire qu'il ne peut pas créer un autre être intelligent ex libre.

Thème $(f)$ : Destin de l'bomme. échec. mal, malice. -

1) Dire trop du destin de l'homme, c'est se satisfaire d'une justice qui serait enclose dans le temps ; c'est (en manichéen) croire au MAL en soi, alors qu'il peut n'être qu'une "privation "; c'est s'apitoyer indûment sur des malheurs qui passent ; c'est cultiver un ressentiment propre à excuser notre paresse et la perte du sens de notre dignité ; c'est refuser de voir les défis où ils sont, dans le temps et au-delà.

2) Dire trop peu du destin de l'homme et du mal, c'est oublier confortablement la souffrance des "autres"; c'est faire un usage prématuré de l'idée que le bien sort souvent du mal ; c'est boucher l'avenir de l'homme ; c'est dire que l'homme n'est pas capable de malice et d'un refus coupable de Dieu.

CONCLUSION GÉNÉRALE de cette dialectique

L'homme se définit pas sa situation médiane, en ce qu'il est, doit penser, dire et faire. La Sagesse qui lui est demandée ne peut résulter que d'un équilibre entre une tension et un consentement (abandon), entre le plaisir et la révolte. Exercée, cette attitude devient "habitus ", seconde nature, force, ( virtus "), qui lui permet de vaincre, de surmonter le non-sens, pour autant toujours que cela est possible, à mesure bumaine.

Université de Sherbrooke 